\title{
ZETA FUNCTIONS IN TRIANGULATED CATEGORIES
}

\author{
V. GULETSKIY
}

\begin{abstract}
We prove 2-out-of-3 property for rationality of motivic zeta function in distinguished triangles in Voevodsky's category DM. As an application, we show rationality of motivic zeta functions for all varieties whose motives are in the thick triangulated monoidal subcategory generated by motives of quasi-projective curves in DM. Joint with a result of P.O'Sullivan it also gives an example of a variety whose motive is not finite-dimensional while the motivic zeta function is rational.
\end{abstract}

\section{Introduction}

Studying objects of a given category we usually wish to have a notion when these objects are reasonable or appropriate to make concrete computations. For example, a set can be measurable, a series can be converging, a vector space can be finite-dimensional, etc. It is then natural to find such a notion for objects in motivic categories, for example, in the category of Chow motives $\mathbf{M}$ or in Voevodsky's triangulated category DM of motives over a field. Maybe the first step in this direction was made by S.Kimura who has introduced the notion of finite-dimensionality for Chow motives and shown its relation to deep problems in algebraic geometry, see [15]. An algebraic counterpart of Kimura's theory was independently worked up by P.O'Sullivan, see an overview in [1].

$Y$. André has shown, [1], [2], that if a Chow motive $M$ is finite-dimensional in the sense of Kimura-O'Sullivan, the corresponding motivic zeta function $\zeta_{M}(t)$ is rational in the ring $K_{0}(\mathbf{M})[[t]]$, where $K_{0}(\mathbf{M})$ is the Grothendieck group of the category $\mathbf{M}$. In particular, zeta function is rational for motives of the abelian type in $\mathbf{M}$, loc.cit. Moreover, recently F. Heinloth proved that for those motives zeta function satisfies a functional equation, [1], answering the question of André.

Recall that the notion of motivic zeta function is naturally connected with many other important topics in different fields of mathematics. It was introduced by M.Kapranov in [14] who proved its rationality and functional equation for smooth projective curves with respect to any motivic measure $\mu$ with the property $\mu\left(\mathbb{A}^{1}\right) \neq 0$. In particular, if $\mu$ counts the number of points of a curve defined over a finite field, then the motivic zeta function is the usual Hasse-Weil zeta function associated with the curve. The rationality of the Hasse-Weil zeta function for all varieties was done by Dwork in [7].

Date: May 2, 2006.

2000 Mathematics Subject Classification. 16E20, 18D10, 19E15, 55 U35.

Key words and phrases. zeta function, motivic measure, triangulated category, finitedimensional motives, triangulated category of motives over a field, homotopy category of motivic spectra, Grothendieck group of a triangulated category, lambda-ring, rationality. 
The main goal of the present paper is to show that rationality of motivic zeta functions can also be considered as a good notion of measurability for motives in Voevodsky's category DM, possibly adding functional equations or something. We will prove that rationality of motivic zeta function has 2-out-of-3 property in distinguished triangles in the triangulated category $\mathbf{D M}^{\prime}$ of geometrical motives over a field. It follows that if $\mathbf{D} \mathbf{M}_{<1}^{\otimes}$ is a thick tensor subcategory in $\mathbf{D} \mathbf{M}^{\prime}$ generated by motives of quasi-projective curves, the motivic zeta function $\zeta_{M}$ is rational for any motive $M$ from $\mathbf{D M}_{<1}^{\otimes}$. In particular, one can get an example of a not finite-dimensional motive whose zeta function is rational. Finally, 2out-of-3 property of rationality of motivic zeta function in $\mathbf{D M}^{\prime}$ leads to a new conjecture: zeta function $\zeta_{M}$ is rational for any motive $M$ in the category $\mathbf{D M}^{\prime}$.

Our approach is homotopical. We use the fact that under mild assumptions the big category DM is equivalent to the homotopy category of an appropriate model monoidal category enriched by simplicial sets. In that sense it can be considered as an abstract stable homotopy category whose monoidal product comes from the model level. Then 2-out-of-3 property for rationality of zeta functions can be proved using the Postnikoff tower constructed in [10]. Actually, we will build two opposite special lambda-structures on the Grothendieck group of the thick triangulated subcategory of compact objects in any homotopy category of a simplicail model monoidal category localized with $\mathbb{Q}$.

\section{Basics on motivic zeta functions}

Let us start with the folowing important example. Assume that $k=\mathbb{F}_{q}$ is a finite field of $q$ elements, let $X$ be an algebraic variety over $\mathbb{F}_{q}$ and let $\# X\left(\mathbb{F}_{q}\right)$ be the number of $\mathbb{F}_{q}$-rational points on $X$. Then the usual Hasse-Weil zeta function $\zeta_{X}$ associated with $X$ can be defined by the formula

$$
\zeta_{X}(t)=\exp \left(\sum_{n=1}^{\infty} \# X\left(\mathbb{F}_{q^{n}}\right) \frac{t^{n}}{n}\right)
$$

After the application of the exponential formula coming from combinatorics, it can be rewritten as

$$
\zeta_{X}(t)=\sum_{n=0}^{\infty} \# \operatorname{Sym}^{n} X\left(\mathbb{F}_{q}\right) t^{n},
$$

where $\operatorname{Sym}^{n} X$ is the symmetric power of $X$, i.e. the quotient of $X^{\times n}$ by the action of the symmetric group.

For any field $k$ let $V$ ar be the category of quasi-projective varieties over $k$. Let, furthermore, $\mathbb{Z}[V a r]$ be the free abelian group generated by isomorphism classes $[X]$ of quisi-projective varieties $X$ over $k$. The Grothendieck group $K_{0}(V a r)$ of the category $V a r$ is, by definition, the quotient of $\mathbb{Z}[V a r]$ by the minimal subgroup containing relations of the type $[X]=[Z]+[X \backslash Z]$ where $Z$ is a closed subvariety in $X$. Then $K_{0}(V a r)$ is naturally a commutative ring with unit and with a product induced by fibered products of varieties over $k$. A motivic measure $\mu$ is a ring homomorphism $\mu: K_{0}(V a r) \rightarrow A$ to any other commutative 
ring $A$. Given $\mu$, for any variety $X$ over $k$ we can consider the corresponding zeta-function

$$
\zeta_{X, \mu}(t)=\sum_{n=0}^{\infty} \mu\left[\operatorname{Sym}^{n} X\right] t^{n}
$$

see [14].

For example, if $\mu$ counts the number of points over a finite field, then $\zeta_{X, \mu}(t)$ is the above Hasse-Weil zeta function of $X$. In that case $\zeta_{X}(t)$ is rational by Dwork's result, [7].

Kapranov proved, see [14, 1.1.9], that the motivic zeta function $\zeta_{X, \mu}(t)$ is rational when $X$ is a smooth projective curve carrying a divisor of degree one, $A$ is a field and the motivic measure $\mu\left(\mathbb{A}^{1}\right)$ of the affine line is not zero. On the other hand, Larsen and Lunts have shown in [16] that there exists a measure $\mu$ built on the base of Hodge numbers $h^{i, 0}$, such that $\zeta_{X, \mu}$ is not rational for a surface $X$ with $h^{2,0} \neq 0$.

Now let $\mathbf{M}$ be the category of Chow motives over $k$ with coefficients in $\mathbb{Q}$. Since $\mathbf{M}$ is a symmetric monoidal additive category, one can construct its Gothendieck ring $K_{0}(\mathbf{M})$ in a standard way, i.e. taking direct sums as sums and tensors products as products in $K_{0}$. In [8] Gillet and Soulé constructed a motivic measure

$$
\mu_{G S}: K_{0}(V a r) \longrightarrow K_{0}(\mathbf{M})
$$

sending a smooth projective variety $X$ to the class of its Chow motive $M(X)$. For any given Chow motive $M$ let

$$
\zeta_{M}(t)=\sum_{n=0}^{\infty}\left[\operatorname{Sym}^{n} M\right] t^{n}
$$

be the corresponding zeta function with coefficients $K_{0}(\mathbf{M})$. By the result of Del Baño and Navarro Aznar, [4],

$$
\mu_{G S}\left[\operatorname{Sym}^{n} X\right]=\left[\operatorname{Sym}^{n} M(X)\right],
$$

whence

$$
\zeta_{M(X)}=\zeta_{X, \mu_{G S}}
$$

for any $X$. If $M$ is a Chow motive which is finite-dimensional in the sense of Kimura-O'Sullivan, then $\zeta_{M}(t)$ is rational, see [1] and [2].

A board generalization can be done as follows. Let $\mathbf{A}$ be any pseudoabeian symmetric monoidal $\mathbb{Q}$-linear category with a monoidal product $\otimes$. Then we have wedge and symmetric powers of objects $X$ in $\mathbf{A}$ as images of the corresponding idempotents in the group algebra of the symmetric group $\Sigma_{n}$ acting on $X^{\otimes n}$. Let $\mathbb{Z}[\mathbf{A}]$ be the free abelian group generated by isomorphisms classes of objects in $\mathbf{A}$, and let $K_{0}(\mathbf{A})$ be the Grothendieck group of the category $\mathbf{A}$, i.e. the quotient of $\mathbb{Z}[\mathbf{A}]$ by the minimal subgroup generated by expressions of type

$$
[X \oplus Y]-[X]-[Y] \text {. }
$$

Clearly, it has a ring structure induced by the monoidal product in $\mathbf{A}$. 
Recall that a lambda-structure on a commutative ring $A$ with unit 1 is a chain of endomorphisms

$$
\lambda^{i}: A \rightarrow A, i=0,1,2, \ldots,
$$

such that $\lambda^{0}(a)=1, \lambda^{1}(b)=r$ and

$$
\lambda^{n}(a+b)=\sum_{i+j=n} \lambda^{i}(a) \lambda^{j}(b)
$$

for all $a$ and $b$ in $A$. It can be also defined as a group homomorphism

$$
\lambda_{t}: A \longrightarrow 1+A[[t]]^{+}
$$

from the additive group of $A$ to the multiplicative group $1+A[[t]]^{+}$of formal power series of type $1+a_{1} t+a_{2} t^{2}+\ldots$ The group $1+A[[t]]^{+}$has a lambda-structure itself, and the lambda-structure on $A$ is called to be special if $\lambda_{t}$ commutes with lambda-operations. Given two lambda structures $\lambda$ and $\sigma$ on the same ring, they are called to be opposite if

$$
1+\sum_{i=1}^{+\infty} \lambda^{i}(a) t^{i}=\left(1+\sum_{i=1}^{+\infty} \sigma^{i}(a)(-t)^{i}\right)^{-1}
$$

for all $a$ in $A$.

Turning back to the category $\mathbf{A}$, wedge and symmetric powers in it give rise to special $\lambda$-structures in the ring $K_{0}(\mathbf{A})$, opposite each other, [11, 4.1]. We will denote these $\lambda$-structures by $\lambda_{+}$for wedge and $\lambda_{-}$for symmetric powers respectively. For example, if $X \in \mathrm{Ob}(\mathbf{A})$ then

$$
\lambda_{+}^{n}[X]=\left[\wedge^{n} X\right]
$$

and

$$
\lambda_{-}^{n}[X]=\left[\operatorname{Sym}^{n} X\right]
$$

Let also

$$
\lambda_{t}^{ \pm}: K_{0}(\mathbf{A}) \longrightarrow 1+K_{0}(\mathbf{A})[[t]]^{+}
$$

be the group homomorphism corresponding to the $\lambda$-structure $\lambda_{ \pm}$. Then for any object $X$ in $\mathbf{A}$ we can define its zeta function $\zeta_{X}(t)$ by the formula:

$$
\zeta_{X}(t):=\lambda_{t}^{-}([X]) \text {. }
$$

If $\mathbf{A}$ is the category of Chow motives $\mathbf{M}$ then we arrive to the above motivic zeta function with respect to the measure constructed by Gillet and Soulé.

Below we are mainly interested in motivic measures $\mu$ which can be factored through $\mu_{G S}$ :

$$
\mu=\tau \circ \mu_{G S}
$$

for some $\tau$. In that case, if we know rationality of $\zeta_{M(X)}$, then we also know rationality of $\zeta_{X, \mu}$.

Any reasonable motivic measure which can be defined in terms of appropriate cohomology groups can be factored through $K_{0}(\mathbf{M})$. For example, given any quasi-projective variety $X$ over $\mathbb{C}$ we define its Hodge numbers $h^{p, q}$ as dimensions of the corresponding bigraded pieces of the mixed Hodge structure on the cohomology with compact support $H_{c}^{p+q}(X, \mathbb{Q})$ of $X$. Then the motivic measure 
sending $X$ to its Hodge polynomial $\sum h^{p, q} u^{p} v^{q}$ can be defined in terms of mixed Hodge realizations. Therefore it factors through $K_{0}(\mathbf{M})$. Another interesting example of a measure factoring through $\mu_{G S}$ can be provided by conductors of I-adic representations over a number field, see [6].

Finally, let us turn to the triangulated setting. Let $\mathbf{T}$ be a triangulated category with shift functor

$$
X \mapsto \Sigma X,
$$

and let $\mathbb{Z}[\mathbf{T}]$ be the free abelian group generated by isomorphism classes of objects in $\mathbf{T}$. Let, furthermore, $S(\mathbf{T})$ be the minimal subgroup in $\mathbb{Z}[\mathbf{T}]$ generated by elements

$$
[Y]-[X]-[Z]
$$

whenever $Y=X \oplus Z$, and let $T(\mathbf{T})$ be the minimal subgroup generated by the same expressions whenever

$$
X \rightarrow Y \rightarrow Z \rightarrow \Sigma X
$$

is a distinguished triangle in $\mathbf{T}$. The quotient $\mathbb{Z}[\mathbf{T}] / S(\mathbf{T})$ is the above usual Grothendieck group denoted now as $K_{0}^{\oplus}(\mathbf{T})$, while the quotient $\mathbb{Z}[\mathbf{T}] / T(\mathbf{T})$ is a more subtle, "triangulated" Grothendieck group of $\mathbf{T}$. Evidently, $K_{0}(\mathbf{T})$ is a quotient of $K_{0}^{\oplus}(\mathbf{T})$ by $T(\mathbf{T}) / S(\mathbf{T})$.

Note that, if $\mathbf{T}$ is a derived category of a nice abelian category $\mathbf{A}$, then $K_{0}(\mathbf{T})$ is isomorphic to $K_{0}(\mathbf{A})$, so that the triangulated $K_{0}$ makes sense.

If we assume that $\mathbf{T}$ is symmetric monoidal, then $K_{0}$ of it is a ring, and the above two subgroups are ideals in $K_{0}$. Assume, furthermore, that $\mathbf{T}$ is $\mathbb{Q}$-linear and pseudoabelian. Then wedge and symmetric powers live in the category. Since $\mathbf{T}$ is additive, $K_{0}^{\oplus}(\mathbf{T})$ has two canonical lambda-structures $\lambda_{ \pm}$by Lemma 4.1 in [11. A crucial question is then whether or not these lambda-structures induce lambda structures on the triangulated group $K_{0}(\mathbf{T})$. The positive answer to this question would have interesting corollaries when applying to zeta functions in the triangulated category of motives over a field.

In the next section we will show an existence of two opposite special lambdastructures in $K_{0}\left(\mathbf{T}^{\prime}\right)$ induced by wedge and symmetric powers in $\mathbf{T}$, where $\mathbf{T}^{\prime}$ is a thick symmetric monoidal subcategory generated by compact objects in any abstract stable homotopy category $\mathbf{T}$.

\section{Main result}

Let $\mathscr{C}$ be a pointed simplicial model monoidal category, let co [12], and let

$$
\mathbf{T}=H_{O}(\mathscr{C})
$$

be the homotopy category of $\mathscr{C}$. Then $\mathbf{T}$ is a triangulated category whose shift functor is induced by the simplicial suspension

$$
\Sigma X=X \wedge S^{1}
$$

in $\mathscr{C}$, loc.cit. Such triangulated category has a symmetric monoidal product $\otimes$ induced by the symmetric monoidal product $\wedge$ of the category $\mathscr{C}$, and it 
can be considered as an abstract prototype for all reasonable stable homotopy categories, see [12].

Since we are interested in the study of wedge and symmetric powers of objects in $\mathbf{T}$, we will assume that $\mathbf{T}$ is $\mathbb{Q}$-localized. If we look on objects in $\mathscr{C}$ as on spectra, then we consider the homotopy category of $\mathbb{Q}$-local spectra.

There are several examples of such triangulated categories arising in algebraic topology and motivic algebraic geometry. The homotopy category of $\mathbb{Q}$-local topological symmetric spectra over a point is just the category of graded $\mathbb{Q}$ vector spaces. This is not interesting. However, the rational stable homotopy theory of $S^{1}$-equivariant spectra is still interesting, see [9].

But the main example for our purposes is the Morel-Voevodsky homotopy category $\mathbf{S H}(X)$ of $\mathbb{Q}$-local motivic symmetric spectra over a Noetherian base scheme $X$, see [13] and [21]. If $X=\operatorname{Spec}(k)$ we will write $\mathbf{S H}$ instead of $\mathbf{S H}(\operatorname{Spec}(X))$.

As it was announced by Morel, [18], if -1 is a sum of squares in the ground field $k$, the category $\mathbf{S H}$ is equivalent to the big category $\mathbf{D M}$ of triangulated motives over $k$.

Let $H_{\mathbb{Z}}$ be the motivic Eilenberg-MacLane spectrum inducing motivic cohomology of schemes over $k$. By the result in [20], the category DM is also equivalent to the homotopy category $\mathrm{Ho}\left(\mathrm{H}_{\mathbb{Z}}-\bmod \right)$ of modules over the ring spectrum $H_{\mathbb{Z}}$ when $k$ is of characteristic zero.

Using these equivalences we can apply any result obtained in $\mathbf{T}=H o(\mathscr{C})$ to the category DM.

Since $\mathbf{T}$ is a homotopy category, it has all coproducts. Then $K_{0}(\mathbf{T})=0$ by Eilenberg swindle. Indeed, let $X$ be an object in $\mathbf{T}$ and let $[X]$ be its class in $K_{0}$. Let

$$
Y=X \coprod x \coprod \ldots
$$

be the coproduct of a countable number of copies of $X$ in $\mathbf{T}$. Then

$$
X \oplus Y=Y,
$$

whence $[X]=0$ in $K_{0}$. Therefore, dealing with $\mathbf{T}=H_{O}(\mathscr{C})$ it is reasonable to work with the thick triangulated subcategory

$$
\mathbf{T}^{\prime}:=\mathbf{T}^{\aleph_{0}}
$$

of compact objects in $\mathbf{T}$, see [19]. For example, if $\mathbf{T}=\mathbf{D M}$ over a field then $\mathbf{T}^{\prime}=\mathbf{D M}^{\prime}$ is nothing but the triangulated category of geometrical motives $D M_{g m}$ over $k$, see [22].

A result is then as follows:

Theorem 1. Wedge and symmetric powers induce two special lambda-structures in the ring $K_{0}\left(\mathbf{T}^{\prime}\right)$, which are opposite each other.

Essentially, the below proof of this theorem is based on the existence of a Postnikoff tower connecting wedge (symmetric) powers of the vertex $Y$ in a 
given distinguished triangle

$$
X \rightarrow Y \rightarrow Y \rightarrow \Sigma X
$$

with wedge (symmetric) powers of two another vertices $X$ and $Z$. Without loss of generality, applying cofibrant replacement, we can assume that both $X$ and $Y$ are cofibrant and the above distinguished triangle is a cofibration triangle, so that $Z=Y / X$ may be considered as a "contraction of $X$ inside $Y$ into a point".

Let $n$ be a natural number and let $K$ be an $n$-cube commutative diagram in $\mathscr{C}$ whose vertices are indexed by $n$-tuples $v=\left\{v_{1}, \ldots, v_{n}\right\}, v_{i}=0,1$, and built up as smash-products $A_{1} \wedge \cdots \wedge A_{n}$ in $\mathscr{C}$ with $A_{j}=X$ if $v_{j}=0$ and $A_{j}=Y$ if $v_{j}=1$. The morphisms of $K$ are smash products of finite collections of copies of the morphism $X \rightarrow Y$ and the identity $X \rightarrow X$, according to vertices in $K$. For example, when $n=2$ the diagram $K$ looks like

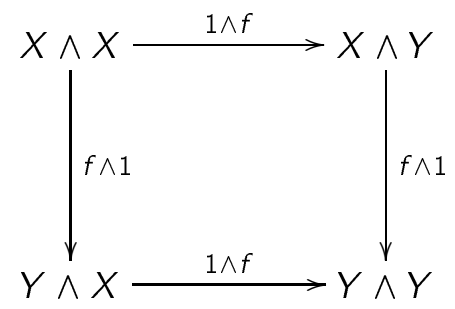

where the objects $X \wedge Y$ and $Y \wedge X$ correspond to the vertices $(0,1)$ and $(1,0)$ respectively.

For any $0 \leq i \leq n$ let $K^{i}$ be the subdiagram in $K$ generated by vertices indexed by $n$-tuples $v$ containing $\leq i$ of units. Then we have the filtration

$$
K^{0} \subset K^{1} \subset \cdots \subset K^{n}=K .
$$

Let

$$
D^{i}=\operatorname{colim} K^{i}
$$

be the colimit of the diagram $K^{i}$ in the category $\mathscr{C}$. Clearly, $D^{0}=X^{\otimes n}$ and $D^{n}=Y^{\otimes n}$. For each $i$ the inclusion $K^{i} \subset K^{i+1}$ induces a morphism

$$
w_{i}: D^{i} \longrightarrow D^{i+1}
$$

on the colimits in $\mathscr{C}$. Let

$$
E^{i+1}=\operatorname{cone}\left(w_{i}\right) \text {. }
$$

Any permutation $\sigma \in \Sigma_{n}$ gives rise to an endomorphism $\Gamma_{\sigma}$ of the distinguished triangle

$$
D^{i} \stackrel{w_{i}}{\longrightarrow} D^{i+1} \longrightarrow E^{i+1} \longrightarrow \Sigma D^{i} .
$$

These $\Gamma_{\sigma}$ are induced by the permutation $\sigma$ on colimits. Considering the idempotents of the group algebra $\mathbb{Q} \Sigma_{n}$ inducing wedge (symmetric) powers in $\mathbf{T}$, we get distinguished triangles

$$
I_{+}^{i} \longrightarrow I_{+}^{i+1} \longrightarrow J_{+}^{i+1} \longrightarrow \Sigma I_{+}^{i}
$$

for the alternated case, and a similar distinguished triangles

$$
I_{-}^{i} \longrightarrow I_{-}^{i+1} \longrightarrow J_{-}^{i+1} \longrightarrow \Sigma I_{-}^{i}
$$


in the symmetric case. It is easy to see that

$$
I_{+}^{0}=\wedge^{n} X, \quad I_{+}^{n}=\wedge^{n} Y
$$

and, similarly,

$$
I_{-}^{0}=\operatorname{Sym}^{n} X, \quad I_{-}^{n}=\operatorname{Sym}^{n} Y .
$$

The key point is that the above cones $C_{ \pm}^{i}$ can be computed by the rule:

$$
J_{+}^{i}=\wedge^{i} Z \otimes \wedge^{n-i} X
$$

and

$$
J_{-}^{i}=\operatorname{Sym}^{i} Z \otimes \operatorname{Sym}^{n-i} X .
$$

The detailed proof of this fact is given in [10].

Now we need a few elementary but useful algebraic observations. Let $M$ be an abelian monoid and let $M^{+}$be the group completion of $M$. The canonical morphism $M \rightarrow M^{+}, m \mapsto[m]$ is universal with respect to all morphisms from $M$ to abelian groups inverting non-zero elements from $M$.

Assume we are given with an equivalence relation $R$ on $M$. We will say that $R$ is additive if the following condition holds: for any two $a, a^{\prime}, b$ and $b^{\prime}$ in $M$, if $\left(a, a^{\prime}\right) \in R$ and $\left(b, b^{\prime}\right) \in R$, then it follows that $\left(a+a^{\prime}, b+b^{\prime}\right)$ is also in $R$. If $R$ is additive then we can construct a quotient additive monoid $M / R$.

Let $R$ be an additive equivalence relation on $M$ and let $R^{+}$be a subgroup in $M$ generated by elements $[a]-\left[a^{\prime}\right]$, such that $\left(a, a^{\prime}\right) \in R$. Then the abelian group $M^{+} / R^{+}$is canonically isomorphic to the abelian group $(M / R)^{+}$because both compositions $M \rightarrow M^{+} \rightarrow M^{+} / R^{+}$and $M \rightarrow M / R \rightarrow(M / R)^{+}$are universal with respect to morphisms $f: M \rightarrow A$ of the monoid $M$ to abelian groups $A$, such that $f(a)=f\left(a^{\prime}\right)$ as soon as $\left(a, a^{\prime}\right) \in R$.

Let $S$ be a set and let $\mathbb{N}[S]$ be a free abelian monoid generated by $S$. Let $\rho$ be a reflexive and symmetric relation on $\mathbb{N}[S]$. If $(a, b) \in \rho$ the we will say that $a$ is elementary $\rho$-equivalent to $b$. Build an additive equivalence relation $\langle\rho\rangle$ on $\mathbb{N}[S]$ generated by $\rho$ as follows: two linear combinations $a$ and $a^{\prime}$ from $\mathbb{N}[S]$ are called to be equivalent if there exist a chain of linear combinations $a_{0}, a_{1}, \ldots a_{n}$, such that $a_{0}=a, a_{n}=a^{\prime}$ and for each $i$ the element $a_{i+1}$ can be obtained from $a_{i}$ by a replacement of a summand in $a_{i}$ by a $\rho$-equivalent summand. For short, let $\mathbb{N}[S] / \rho$ be the quotient of $\mathbb{N}[S]$ by $\langle\rho\rangle$ and $\rho^{+}=\langle\rho\rangle^{+}$. Certainly, $(\mathbb{N}[S])^{+}=\mathbb{Z}[S]$. From the previous observation we have:

$$
(\mathbb{N}[S] / \rho)^{+}=\mathbb{Z}[S] / \rho^{+} .
$$

For example, let $\mathbf{A}$ be an additive category, $\mathbb{N}[\mathbf{A}]:=\mathbb{N}[\mathrm{Ob}(\mathbf{A})]$ and let $\sigma$ be the set of pairs $([X \oplus Y],[X]+[Y])$ and their transposes. We see that $\mathbb{Z}[\mathbf{A}] / \sigma^{+}$ is the additive Grothendieck group $K_{0}^{\oplus}(\mathbf{A})$ of $\mathbf{A}$. The above isomorphism shows then that $K_{0}^{\oplus}(\mathbf{A})$ can be also described as a completion of the monoid $\mathbb{N}[\mathbf{A}] / \sigma$.

In the triangulated situation we have the following. Let $\mathbf{T}$ be a triangulated category, $\mathbb{N}[\mathbf{T}]:=\mathbb{N}[\mathrm{Ob}(\mathbf{T})]$ and let $\Delta$ be the set of pairs $([Y],[X]+[Z])$, where $X Y Z$ is a distinguished triangle, and their transposes. Again we see that $\mathbb{Z}[\mathbf{T}] / \Delta^{+}$is the triangulated Grothendieck group $K_{0}(\mathbf{T})$ and the above 
isomorphism shows then that it can be also described as a completion of the monoid $\mathbb{N}[\mathbf{T}] / \Delta$.

In order to finish the proof of Theorem 11 we will consider the case of wedge powers only. The symmetric case is analogous.

For any object $X$ in $\mathbf{T}$ let $[X]$ be its class in the Grothendieck group, $K_{0}^{\oplus}$ or $K_{0}$. We have a countable set of maps

$$
\lambda^{n}: K_{0}^{\oplus}(\mathbf{T}) \longrightarrow K_{0}^{\oplus}(\mathbf{T}), n=0,1,2, \ldots,
$$

such that

$$
\lambda^{n}[X]=\left[\wedge^{n} X\right]
$$

for any $X$ in $\mathbf{T}$, and the value of $\lambda^{n}$ on a sum $[X]+[Y]$ is determined by the Künneth rule. These are the $\lambda$-operations considered in [11]. In order to define $\lambda$-operations by the same rule in $K_{0}(\mathbf{T})$ we need only to show that the above maps $\lambda^{i}$ respect also the subgroup $T(\mathbf{T})$. Since $K_{0}(\mathbf{T})=(\mathbb{N}[\mathbf{T}] / \Delta)^{+}$, it is enough to show that if two linear combinations $a$ and $b$ are elementary $\Delta$-equivalent, then $\lambda^{n} a$ is $\Delta$-equivalent to $\lambda^{n} b$.

Without loss of generality we can assume that $a=[Y], b=[X]+[Z]$ and we have a distinguished triangle

$$
X \longrightarrow Y \longrightarrow Z \longrightarrow \Sigma Z
$$

in $\mathbf{T}$. For each $n$ let

$$
\begin{gathered}
I_{+}^{i} \longrightarrow I_{+}^{i+1} \longrightarrow J_{+}^{i+1} \longrightarrow \Sigma I_{+}^{i}, \\
i=0,1, \ldots, n-1,
\end{gathered}
$$

be the Postnikoff system as above. Let

$$
a_{i}=\left[I_{+}^{i}\right], \quad c_{i}=\left[J_{+}^{i}\right], \quad x_{i}=\left[\wedge^{i} X\right], \quad y_{i}=\left[\wedge^{i} Y\right] \quad \text { and } \quad z_{i}=\left[\wedge^{i} Z\right]
$$

for all non-negative integers $i$. The above Postnikoff tower can be rewritten in terms of the ring $K_{0}(\mathbf{T})$ as follows:

$$
\begin{gathered}
a_{0}=x_{n}, \quad a_{n}=y_{n}, \\
c_{i}=z_{i} x_{n-i}, \quad i=0,1, \ldots, n,
\end{gathered}
$$

and

$$
a_{i+1}=a_{i}+c_{i+1}, \quad i=0,1, \ldots, n-1,
$$

whence we get:

$$
y_{n}=\sum_{i=0}^{n} z_{i} x_{n-i}
$$

in the ring $K_{0}(\mathbf{T})$, where $y_{n}=\lambda^{n}[Y], x_{0}=1$ and $z_{0}=1$. It means that the element $\lambda^{n}([X]+[Z])$ is $\Delta$-equivalent to the element $\lambda^{n}[Y]$ in the ring $K_{0}^{\oplus}$, as desired. 
Let $\Lambda^{i}$ be the $i$-th operation of the canonical lambda-structure on $\left.1+K_{0}\left(\mathbf{T}^{\prime}\right)[[t]]\right]^{+}$. The precompositions of the two paths $\Lambda^{i} \lambda_{t}$ and $\lambda_{t} \lambda^{i}$ in the diagram

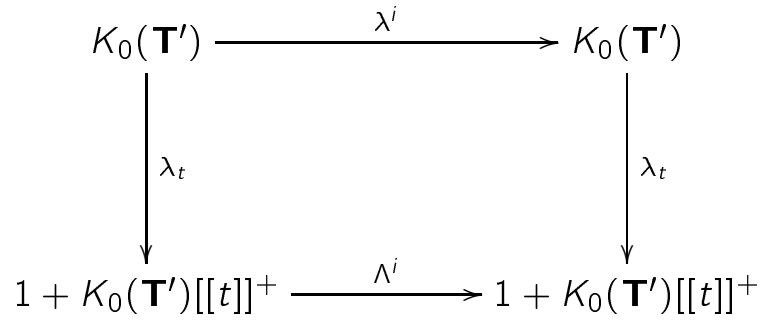

with the surjective homomorphism

$$
K_{0}^{\oplus}\left(\mathbf{T}^{\prime}\right) \longrightarrow K_{0}\left(\mathbf{T}^{\prime}\right)
$$

coincide because the $\lambda$-structure on $K_{0}^{\oplus}$ is special. Therefore the diagram is commutative, i.e. the induced $\lambda$-structure on the $K_{0}$-level is special as well.

By the same argument, the lambda-structure on $K_{0}$ defined by wedge powers is opposite to the $\lambda$-structure defined by symmetric powers.

\section{Some applications}

A formal power series $\xi(t)$ in variable $t$ with coefficients in a commutative ring $A$ is called rational, if there exists two polynomials $a(t)$ and $b(t)$ in $A[t]$, such that $a \xi=b$ in $A[[t]]$.

For any element $a \in K_{0}\left(\mathbf{T}^{\prime}\right)$ let $\zeta_{a}(t)=\lambda_{t}^{-}(a)$ be the corresponding zeta function of $a$. If $a=[X]$ for some object $X$ in $\mathbf{T}^{\prime}$, then we write $\zeta_{X}$ instead of $\zeta_{a}$. The suspension $\Sigma: \mathbf{T} \rightarrow \mathbf{T}$ induces an involution $a \mapsto-a$ on $K_{0}\left(\mathbf{T}^{\prime}\right)$. Theorem 1 1 gives $\zeta_{-a}=\zeta_{a}^{-1}$ for any element $a$ in $K_{0}$. In particular, $\zeta_{\Sigma X}=\left(\zeta_{X}\right)^{-1}$ for any object $X$ in $\mathbf{T}^{\prime}$. It follows that the suspension does not change rationality of the corresponding zeta function. Also we have:

Corollary 2. Let $X \rightarrow Y \rightarrow Z \rightarrow \Sigma X$ be a distinguished triangle in $\mathbf{T}$. If two out of three zetas $\zeta_{X}, \zeta_{Y}$ and $\zeta_{Z}$ are rational functions, then the third zeta function is also rational.

Proof. Since $X Y Z$ is a distinguished triangle, $[Y]=[X]+[Z]$ in $K_{0}(\mathbf{T})$. Since we work with zeta-functions induced by $\lambda$-structures in $K_{0}$,

$$
\zeta_{Y}=\zeta_{X} \cdot \zeta_{Z}
$$

whence the proof.

Assume now that either $\operatorname{char}(k)=0$ or -1 is a sum of squares in $k$. In that case, as we have seen in Section 3 the category DM can be interpreted as a category $\mathbf{T}=H_{O}(\mathscr{C})$ via the equivalence with either the motivic stable category $\mathbf{S H}$ or with the homotopy category of modules over the motivic Eilenberg-MacLane spectrum. Therefore, Theorem [1 can be applied to the category $\mathbf{D M}^{\prime}$ as well. For any motive $M \in \mathbf{D M}^{\prime}$ let $\zeta_{M}(t)$ be the corresponding motivic zeta function of $M$. 
The advantage of Theorem 1 is that it extends the range of varieties whose motivic zeta function is rational. Indeed, let $\mathbf{D} \mathbf{M}_{<1}^{\otimes}$ be a thick symmetric monoidal subcategory in DM generated by motives of quasi-projective curves over $k$.

Corollary 3. For any motive $M$ in $\mathbf{D M}_{\leq 1}^{\otimes}$ its zeta function $\zeta_{M}(t)$ is rational.

Proof. The motive $M(X)$ of a quasi-projective curve $X / k$ is finite-dimensional in the sense of Kimura-O'Sullivan, see [10] or [17]. Therefore, $\zeta_{M(X)}$ is strictly rational by André's result, see [2, 13.3.3.1]. If $M$ is in $\mathbf{D M}_{\leq 1}^{\otimes}$, then its class $[M]$ belongs to a subring in $K_{0}\left(\mathbf{D M}^{\prime}\right)$ generated by classes of motives of quasiprojective curves.

Given a $\lambda$-structure on a ring $A$, if $\zeta_{a}$ and $\zeta_{b}$ are rational for two elements $a$ and $b$ in $A$, then $\zeta_{a+b}$ is also rational. If we assume, furthermore, that this lambda-structure is special, then $\zeta_{a b}=\zeta_{a} \star \zeta_{b}$ where $\star$ is the product in the lambda-ring $1+A[[t]]^{+}$. At the same time, for any two elements $f(t)$ and $g(t)$ in $1+A[[t]]^{+}$if $f$ and $g$ are ratio of polynomials then so is the product $f \star g$.

Since the lambda-structure on $K_{0}\left(\mathbf{D M}^{\prime}\right)$ is special, rationality of zeta functions is closed under sums and products of elements in $K_{0}\left(\mathbf{D M}^{\prime}\right)$, whence the result.

Corollary 2 says that motivic zeta function has 2-out-of-3 property in distinguished triangles in $\mathbf{D M}^{\prime}$. This correlates with Lemma 3.1 in [16] via Gyzin distinguished triangles in the category $\mathbf{D M}^{\prime}$. In general, there are several canonical distinguished triangles in DM each of which gives new examples of varieties whose motivic zeta function is rational.

Example 4. Let $X$ be a $\mathrm{K} 3$ surface over $\mathbb{C}$, such that $M(X)$ is in $\mathbf{D M}_{<1}^{\otimes}$. This is so if $X$ is, for example, of Kummer type or a Weil quartic in $\mathbb{P}^{3}$. Then $\zeta_{X}$ is rational by Corollary 3 . As it was shown by O'Sullivan, [17], there exists a Zariski open $U$ in $X$, such that the motive $M(U)$ is not finite-dimensional. However, the function $\zeta_{U}$ is rational. Indeed, the complement $Z=X-U$ is a union of curves. The motive of any quasi-projective curve is finite-dimensional, whence rationality of $\zeta_{Z}$. Since $\zeta_{X}$ is rational, applying Corollary 2 to the Gyzin distinguished triangle in $\mathbf{D M}$ associated with the pair $X, U$ we see that $\zeta_{U}$ is rational.

Example 5. Let $X=X_{1} \cup \cdots \cup X_{n}$ be a union of quasi-projective surfaces whose zeta functions $\zeta_{X_{i}}$ are rational. Then $\zeta_{X}$ is rational. Indeed, if $n=2$, we apply Corollary 2 and rationality of zeta functions of quasiprojective curves to the corresponding Mayer-Vietoris distinguished triangle in DM. If $n>2$, we just write down an appropriate Postnikoff tower and apply Corollary 2 several additional times.

Example 6. Let $X$ be a quasi-projective variety over $k$, let $Z$ be a closed subvariety in $X$ and let $f: X^{\prime} \rightarrow X$ be a blow up of $X$ in $Z$. Assume that $\zeta_{Z}$ is rational. Then we use the corresponding blow up distinguished triangle in DM 
in order to show that $\zeta_{X}$ is rational if and only if $\zeta_{X^{\prime}}$ is rational. Of course, if all appearing varieties are smooth projective, then the same claim follows from the result in [11] and Manin's motivic formula for blow ups in the category of Chow motives over $k$.

Example 7. Let $C$ be a smooth projective curve, let $Y$ be a quotient of $C \times C \times C$ by the cyclic action of $\mathbb{Z} / 3$, and let $X$ be resolution of singularities of $Y$. Then $X$ is a smooth projective threefold whose motive is in $\mathbf{D} \mathbf{M}_{\leq 1}^{\otimes}$ and the motivic $\zeta_{X}$ is rational.

Remark 8. By recent result of Bondarko, [5], $K_{0}(\mathbf{M})$ is isomorphic to the triangulated $K_{0}\left(\mathbf{D M}^{\prime}\right)$ if we consider both categories over a field of characteristic zero. Most probably this isomorphism also gives the two above canonical lambda-structures in Voevodsky's category $\mathbf{D M}^{\prime}$. However, Theorem 1 allows to work with lambda-structures in the 2-functor $\mathbf{S H}(-)$ on schemes in general. So, on the one hand, we can use $\mathbf{S H}(-)$ in order to build new varieties whose motivic zeta functions are rational, on the other hand, we can use Bondarko's isomorphism in order to factor interesting motivic measures through $K_{0}\left(\mathbf{D M}^{\prime}\right)$.

Example 9. Assume we are given with a nice motivic measure $\mu$ which factors through $K_{0}(\mathbf{M})$, say $\mu$ is made by Hodge polynomials of varieties over $\mathbb{C}$ or by conductors of I-adic representations for étale cohomology of varieties over $\mathbb{Q}$. Since $K_{0}(\mathbf{M})$ is naturally isomorphic to $K_{0}\left(\mathbf{D M}^{\prime}\right)$, we can also factor $\mu$ through $K_{0}\left(\mathbf{D M}^{\prime}\right)$ and apply Corollary 3 . Then we have that $\zeta_{X, \mu}$ is rational for any variety $X$, such that $M(X)$ is in $\mathbf{D} \mathbf{M}_{\leq 1}^{\otimes}$.

Remark 10. Since $\mathbf{D M}^{\prime}$ is generated by $\mathbf{M}$, Theorem 1 shows that if $\zeta_{M}$ is rational for all Chow motives over $k$, then $\zeta_{M}$ is rational for all motives in $\mathbf{D M}^{\prime}$. Let $\eta$ be the generic point of the affine line $\mathbb{A}^{1}$, and let 0 be a closed point on it. Let

$$
\Psi: \mathbf{D M}^{\prime}(\eta) \longrightarrow \mathbf{D M}^{\prime}(0)
$$

be the nearby cycle motivic functor constructed by J.Ayoub in [3]. The category $\mathbf{D M}^{\prime}$ is generated by motives of type $\Psi M(X)$ where $X$ is a smooth hypersurface in a projective space over $\eta$, loc.cit. Therefore, in order to show rationality of motivic zetas for all motives in $\mathbf{D M}^{\prime}$ it is enough to show that $\zeta_{X}$ is rational for a smooth projective hypersurface $X$ in $\mathbb{P}^{n}$ over $\eta$. The standard approach is as follows. Let $\mathscr{X} \rightarrow \mathbb{P}^{N}$ be a universal family of smooth hypersurfaces of degree $d$ over a field. Draw a line $\mathbb{A}^{1}$ in the parameter space $\mathbb{P}^{N}$ through a smooth hypersurface $X$ and a Weil type hypersurface $W$. Restricting the universal family on $\mathbb{A}^{1}$ we get a smaller family $\mathscr{Y} \rightarrow \mathbb{A}^{1}$ which deforms $X$ into $W$. The motivic $\zeta_{W}$ is rational, of course. Then one can try to deform rationality of $\zeta_{W}$ to rationality of $\zeta_{X}$ using the homomorphism

$$
K_{0} \Psi: K_{0}\left(\mathbf{D M}^{\prime}(\eta)\right) \longrightarrow K_{0}\left(\mathbf{D M}^{\prime}(0)\right) \text {. }
$$

This is, actually, nothing else but a $K_{0}$-simplification of Ayoub's approach to Schur-finiteness of $\mathbf{D} \mathbf{M}^{\prime}$. Note that if $M$ is a Chow motive over $\eta$, then we can 
use the nice properties of the functor $\psi$ established in [3] in order to compute $K_{0} \Psi[M]$ in terms of the usual Chow specialization of the motive $M$ to 0 .

Acknowledgements. I thank Joseph Ayoub for several useful discussions along the theme of this paper. The work was supported by NSF Grant DMS0111298. I am grateful to the Institute for Advanced Study at Princeton for the support and hospitality in 2005-2006 academical year.

\section{References}

[1] Y. Andre. Motifs de dimension fini. Séminaire Bourbaki, Mars 2004, Exposés 929

[2] $Y$. Andre. Une introduction aux motifs (motifs purs, motifs mixtes, périodes). Panoramas et Synthèses, 17. Société Mathématique de France, Paris, 2004

[3] J. Ayoub. The motivic vanishing cycles and the conservation conjecture. To appear in the Proceedings of the conference in ocassioan of 75 birthday of Jacob Murre, Leiden 2004

[4] R.S. del Baño, V. Navarro Aznar. On the motive of a quotient variety. Collect. Math. 49 (1998) no. 2-3, $203-226$

[5] M. Bondarko. Weight filtrations and motivic descent spectral sequence for differential graded realizations of the Voeovodsky motives; enhancement and truncations for the category of motives. Preprint, math.AG/0601713

[6] J. Denef, F. Loeser. Geometry on arc spaces of algebraic varieties. European Congress of Mathematics, Vol. I (Barcelona, 2000), 327 - 348, Progr. Math., 201, Birkhäuser, Basel, 2001

[7] B. Dwork. On the rationality of the zeta function of an algebraic variety. Amer. J. Math. $82(1960) 631-648$

[8] H. Gillet, C. Soulé. Descent, motives and K-theory. J. Reine Angew. Math. 478 (1996), $127-176$

[9] J. P. C. Greenlees. Rational $S^{1}$-equivariant stable homotopy theory. Mem. Amer. Math. Soc. 138 (1999), no. 661

[10] V. Guletskiř. Finite dimensional objects in distinguished triangles. To appear in Journal of Number Theory.

[11] F. Heinloth. A note on functional equations for zeta functions with values in Chow motives. Preprint, math.AG/0512237

[12] M. Hovey. Model categories. Mathematical Surveys and Monographs, 63. American Mathematical Society, Providence, RI, 1999

[13] J.F. Jardine. Motivic symmetric spectra. Documenta Mathematica (2000) 445 - 553

[14] M. Kapranov. The elliptic curve in the S-duality theory and Eisenstein series for KacMoody groups. Preprint, math.AG/0001005

[15] S.-I. Kimura. Chow groups are finite dimensional, in some sense. Math. Ann. 331 (2005), no. $1,173-201$

[16] M. Larsen, V. Lunts. Rationality criteria for motivic zeta functions. Compos. Math. 140 (2004) no. 6, 1537 - 1560

[17] C.Mazza, Carlo Schur functors and motives. K-Theory 33 (2004) no. 2, 89 - 106

[18] F. Morel. On the motivic $\pi_{0}$ of the sphere spectrum. Axiomatic, enriched and motivic homotopy theory, 219 - 260, NATO Sci. Ser. II Math. Phys. Chem., 131, Kluwer Acad. Publ., Dordrecht, 2004

[19] A. Neeman. Triangulated Categories. Annals of Math. Studies, vol.148. Princeton University Press (2001)

[20] O.Röndigs, P.Østvær. Modules over Motivic Cohomology. Preprint, see Röndigs' homepage

[21] V. Voevodsky. $\mathbb{A}^{1}$-homotopy theory. Doc. Math. (1998), ICM 1998, Berlin, 417 - 442. 
[22] V. Voevodsky. Triangulated categories of motives over a field. In: V.Voevodsky, A. Suslin and E. Friedlander. Cycles, Transfers and Motivic Cohomology Theories. Annals of Math. Studies, 143. P.U.P. Princeton, N.J., U.S.A.

E-mail: guletski@ias.edu 